\title{
THE STELLAR POPULATION OF THE INNER 200 PARSECS
}

\author{
R. MICHAEL RICH* \\ Department of Astronomy, Columbia University \\ 538 West 120th Street, New York, NY 10027, USA
}

\begin{abstract}
The central $200 \mathrm{pc}$ of the Galaxy is obscured by 5-30 visual magnitudes of extinction and therefore may be studied only in the infrared. The central parsec consists of a mostly red star cluster (IRS 16 ) and a cluster of $\approx 10 \mathrm{HeI}$ Wolf-Rayet or Of-like stars; this cluster is the best evidence for star formation in this region. The central $5 \mathrm{pc}$ has an extended giant branch (to $M_{b o l}=-6$ ) and long period variables. These stars are not extraordinarily bright; in the central 200 pc luminous asymptotic giant branch (AGB) stars $\left(M_{b o l}<-5\right)$ concentrate toward the nucleus in a distribution more flattened than the general light. Candidates for relatively young objects include a rapidly rotating population of high outflow velocity $\mathrm{OH} / \mathrm{IR}$ stars. The AGB luminosity function in the central $200 \mathrm{pc}$ resembles that recently found in M32 and M31 bulge fields. Depending on how one interprets the extended giant branch, the population may be intermediate age with some ongoing star formation, or mostly old and metal rich, with star formation confined to the nucleus.
\end{abstract}

\section{Introduction}

During the July, 1945 dark run Stebbins and Whitford (1947) began to search for the Galactic nucleus by drift scanning across the Sagittarius region using a $\mathrm{CsO}$ photocell and ballistic galvanometer attached to the 60 -inch telescope on Mt. Wilson. They were first in observing the central 200 parsecs, finding a central bulge-like stellar population but not uncovering the nucleus; they noted (with foresight) that a search for the nucleus would likely be more successful at $2 \mu \mathrm{m}$. Success arrived some 22 years later, when Becklin \& Neugebauer (1968) discovered the central star cluster at $2.2 \mu \mathrm{m}$, comparing its surface brightness profile to that of the M31 nucleus.

Since its discovery, the Galactic center has been revealed as the site of tremendous activity, possibly an active galactic nucleus in its own right. This review does not address those properties; rather, recent conference

* Alfred P. Sloan Foundation Fellow. 
volumes (see below) address this wide range of complex activity. The aim of this article is to review the properties of and define what we need to learn about the relatively neglected stellar population of the inner 200 pc, or $1.4^{\circ}$ (for $R_{0}=8 \mathrm{kpc}$ ). This region of the bulge lies deep within the zone of obscuration, suffering 10-30 mags of visual extinction. Figure 1a shows a composite infrared-image of the central region (Gatley et al. 1989). The penetrating power of infrared is great, but is still blocked by the heaviest dust clouds. The high extinction of the region is shown in Figure 1b, from Catchpole, Whitelock, \& Glass (1990); at $140 \mathrm{pc} / \mathrm{deg}$ $\left(R_{0}=8 \mathrm{kpc}\right)$ one sees that entire inner $200 \mathrm{pc}$ cannot be studied in the optical.

Figure 2 shows Gatley's new emission line images of the center, revealing some of the remarkable structures arising from the activity there; while not the subject of this review, I include this image to remind the reader of the special conditions at the center.

The following section discusses the population of the central parsec, where activity and possible star formation generate conditions of such intensity that the properties of the stellar atmospheres are affected. $\S 3$ examines the stellar content and distribution of the inner $200 \mathrm{pc}$. We explore similar populations in the local group in $\S 4$ : M32 and the bulge of M31. Finally, we try to apply what we have learned to understanding the formation of the bulge.

This review concerns only the stellar population, and makes no efforts to address the source of the central activity or the existence of a black hole there. These issues are addressed in IAU Symp. 136 (Morris, (ed.) 1989), AIP Conf. 155 (Backer (ed.) 1987) and by Genzel \& Townes (1987). Other aspects of the population are addressed in the recent volume edited by Blitz (1992). This article only addresses dynamics as they concern the stellar populations, not the central mass distribution. Recent efforts on a dynamical models include those of Lindquist et al. (1992) and Kent (1992).

\section{The Central Parsec}

Within this region, a source known as IRS 16 has been resolved (de Poy et al. 1991; Eckart et al. 1992) and is the best candidate for the stellar nucleus; a cluster of late-type stars. It is $1^{\prime \prime}$ distant from $\operatorname{SgrA} A^{*}$, the nonthermal source that is the best black hole candidate, not an unusual circumstance given that the nucleus of M31 is not on centered on the bulge light. Sellgren's (1989) baseline review on the central star cluster discusses the basic properties, including her discovery (Sellgren et al. 

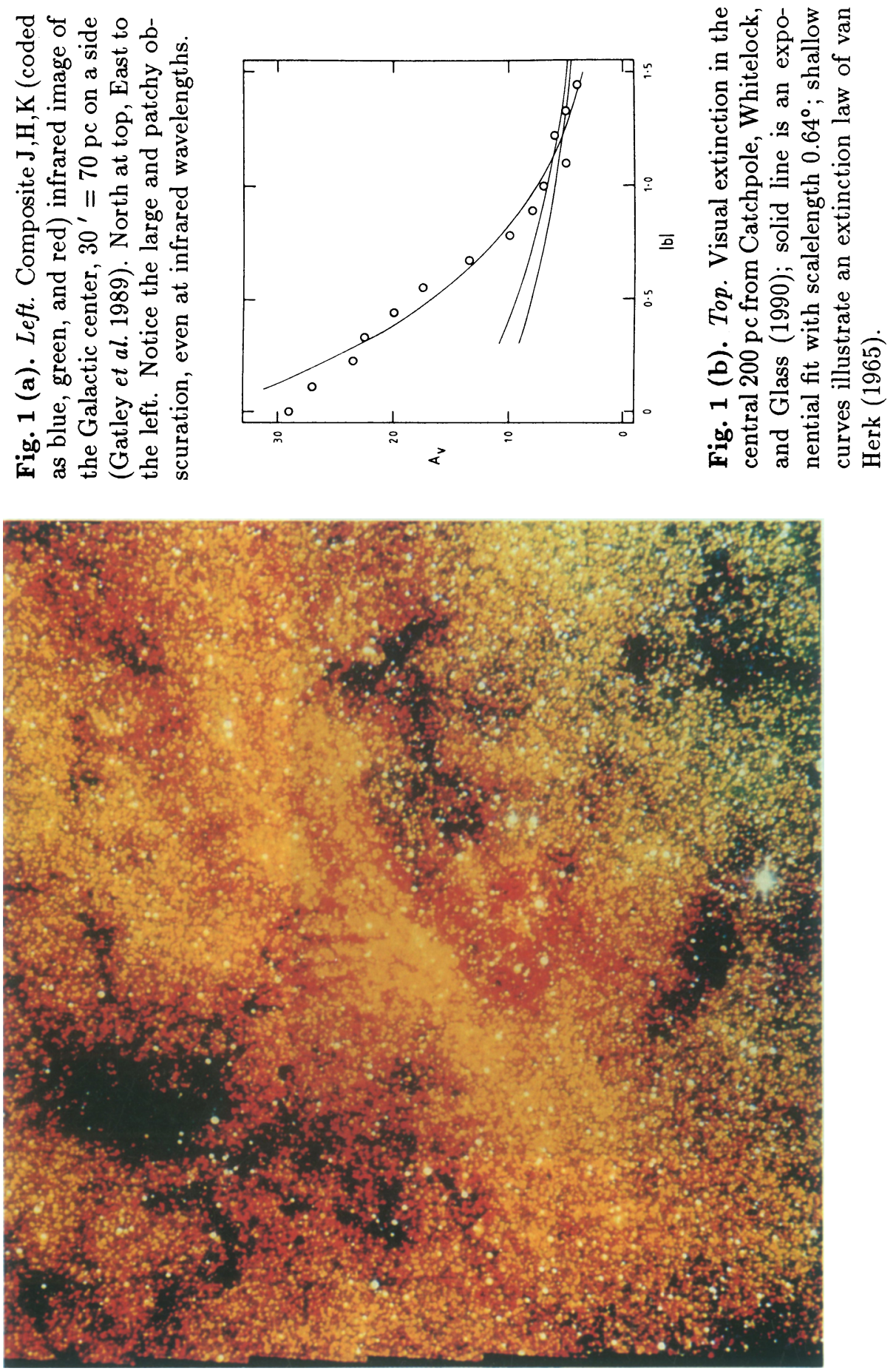


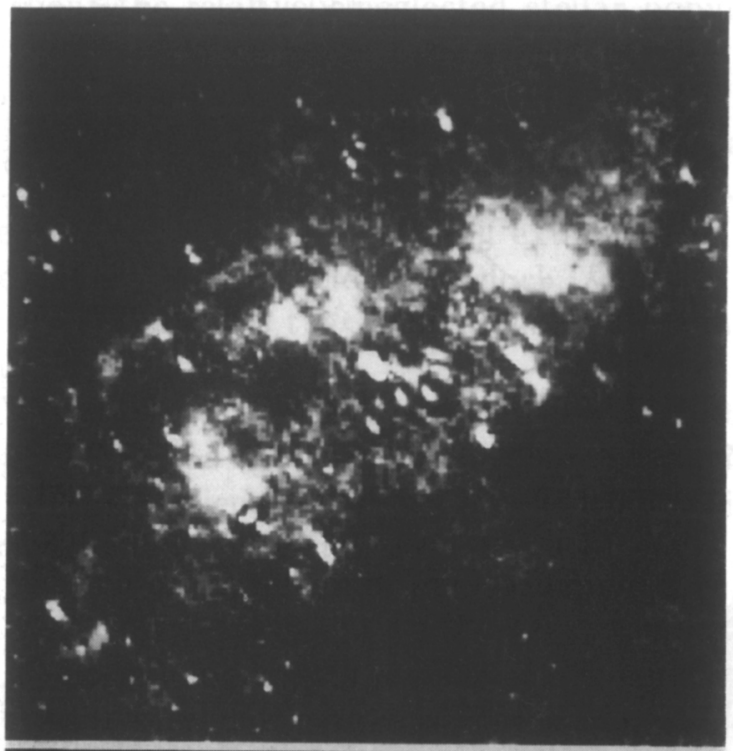

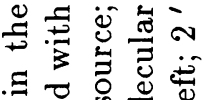

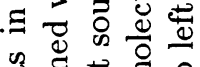

丞.

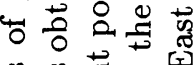

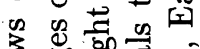

3.0.

$>$ 兘完

उ。

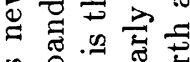

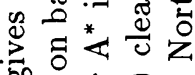

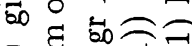

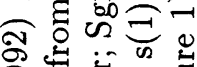

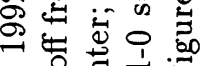

3 80 피

0. $\Xi \geq . \Xi$

菏

苛

$\$ \frac{0}{3} \infty$

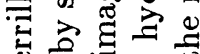

을 至

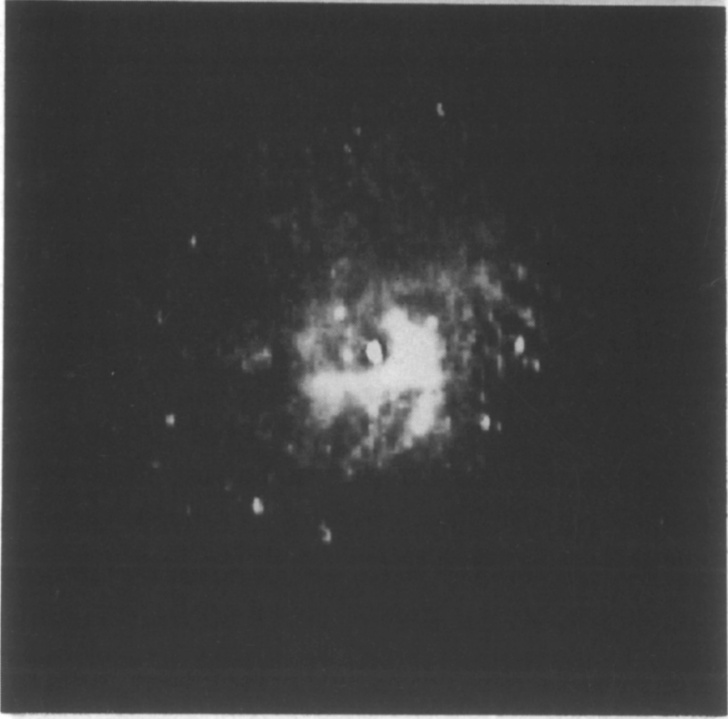

$\sum$ 芯 范

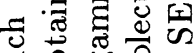

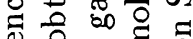

๑ $\doteq \pm$.

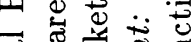

ชี

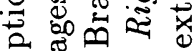

$\bigcirc \stackrel{\square}{0} \dot{0} 0$

.

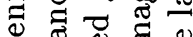

:

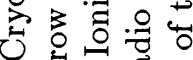

茫芯

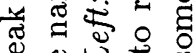

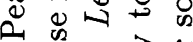

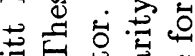

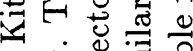

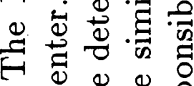

$\circlearrowleft \stackrel{\Theta}{\Theta}$

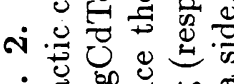

ம) 
1990) that stars within the central pc have weak $2.3 \mu \mathrm{m} \mathrm{CO}$ bands. It is interesting to note that for $R_{0}=8 \mathrm{kpc}$ the central parsec subtends $24^{\prime \prime}$; it may be studied in great detail from the ground, except for the $\approx 30 \mathrm{~V}$ mag of extinction toward this line of sight.

It is in the central pc that we find the best candidates for very young luminous stars; the only direct evidence in this region for star formation. Allen, Hyland, \& Hillier (1990) and Krabbe et al. (1991) find a cluster of He I emission-line stars interpreted to be either Of or Wolf-Rayet. It is significant that star formation and the central star cluster may now account for the total output of far-infrared emission from the center $(\approx$ $10^{7} L_{\odot}$ ), a credible alternative to the proposal (cf. Gatley, 1987) that a central engine must account for this emission. It is interesting that the He I star cluster is much more extended than the central portion of IRS 16. While line imaging and spectroscopy of the He I stars looks convincing, their certification as genuine young stars remains controversial; Morris (1992) suggests that the apparent stars may actually be compact objects with their luminosity from an accretion process.

Some combination of this star cluster and $\operatorname{SgrA}^{*}$ creates a wind strong enough to blow the atmosphere of IRS 7 (the only certain supergiant) into a spectacular comet-like tail (Yusef-Zadeh \& Morris, 1991). This wind may ablate the atmospheres of the giants in the central pc, or the blue star cluster might dissociate the $\mathrm{CO}$, either of which could cause the decreased $\mathrm{CO}$ linestrength seen in the giants. The drop in $\mathrm{CO}$ might also be due to the high stellar density of $\approx 10^{6} M_{\odot} \mathrm{pc}^{-3}$; encounters might strip the atmospheres of half the giants in the central $0.4 \mathrm{pc}$ (Phinney, 1989). On top of the reddening, these effects make it risky to use observed colors and luminosities to infer the age of the red stars in the central pc.

The key question about the population of the central parsec applies to the global population as well: we cannot easily distinguish between a population of intermediate age stars of solar abundance, and possible peculiar evolution of super metal rich stars. Stars of high luminosity are present, but it is hard to tell if they are bright because they are young, or metal rich.

In summary, the central region consists of an unresolved $\left(<1^{\prime \prime}\right)$ red star cluster (IRS16) which is likely the stellar nucleus and is offset from $\mathrm{SrgA}^{*}$, the nonthermal radio source. The central parsec also contains an extended cluster of luminous HeI stars which can by themselves account for nearly all of the emergent luminosity of the central region. There is evidence for $\mathrm{HeI}$ emission (hence possibly star formation) centered on the 
IRS16 cluster. The late-type stars alone account for $\approx 2 \times 10^{6} L_{\odot}$ in the central pc, or $10 \%$ of the total luminosity.

\subsection{THE LATE-TYPE STARS}

The high reddening makes it difficult to measure accurate colors and luminosities to compare the central stars with known populations like the outer bulge. Haller (1992) attacks this problem by measuring both JHK photometry and variability of stars in the central $5^{\prime} \times 5^{\prime}=6 \mathrm{pc}$; the reddest stars are LPV (long period variable) candidates. Figure 3 shows a mosaic image constructed by Haller and Rieke in which some 1000 stars are found, including 59 variable star candidates. Assuming that $L_{T O T} \approx$ $10^{7} L_{\odot}$ (Becklin \& Werner, 1982), we may use the Fuel Consumption Theorem (Renzini \& Buzzoni, 1986) to calculate the expected number of Miras, assuming a lifetime of $2 \times 10^{5} \mathrm{yr}$. One expects to find $\approx 40$ Miras in the central few pc. Adopting a lifetime of $1.3 \times 10^{6} \mathrm{yr}$ per magnitude of evolution for AGB stars, $10^{3}$ AGB stars should be found in the central region; both predictions agree with Haller's numbers. The number of luminous AGB stars is consistent with the total luminosity: they are not merely a trace population.

There are two baseline studies of critical importance in analyzing this population: Frogel \& Whitford (1987); FW87 (the Baade's Window M giants) and Whitelock, Feast \& Catchpole (1991); WFC91 on LPV's selected from the IRAS database. Late $\mathrm{M}$ giants in the bulge first isolated by Blanco (1965) comprise the FW87 optically selected sample, while WFC91 selected a set of cool stars from the IRAS Point Source Catalog with $0.7<f_{25} / f_{12}<2.0$. The work of van der Veen \& Habing (1990) completes the picture, addressing the properties of the rare $\mathrm{OH} / \mathrm{IR}$ stellar population in the bulge.

Figure 4a shows an effort at normalizing the FW87 and Haller (1992) luminosity functions based on $L_{T O T}$ for the stellar population using a modulus of 14.2 to the center (current estimates favor a modulus $0.3 \mathrm{mag}$ larger, hence bolometric magnitudes 0.3 brighter). Figure $4 \mathrm{~b}$ shows that the actual number of luminous stars is large and that the variables are consistent with known properties of Miras. This galactic center population does not exist in isolation, however. Lindqvist et al. (1992) discover numerous $\mathrm{OH} / \mathrm{IR}$ stars there, and a population of luminous stars is found to exist over the entire inner $200 \mathrm{pc}$.

The population of the central $5 \mathrm{pc}$ is not extraordinary; the long period variable star candidates have the colors and luminosities of Miras from the WFC91 sample, which itself lies 1000 pc from the nucleus. Haller 


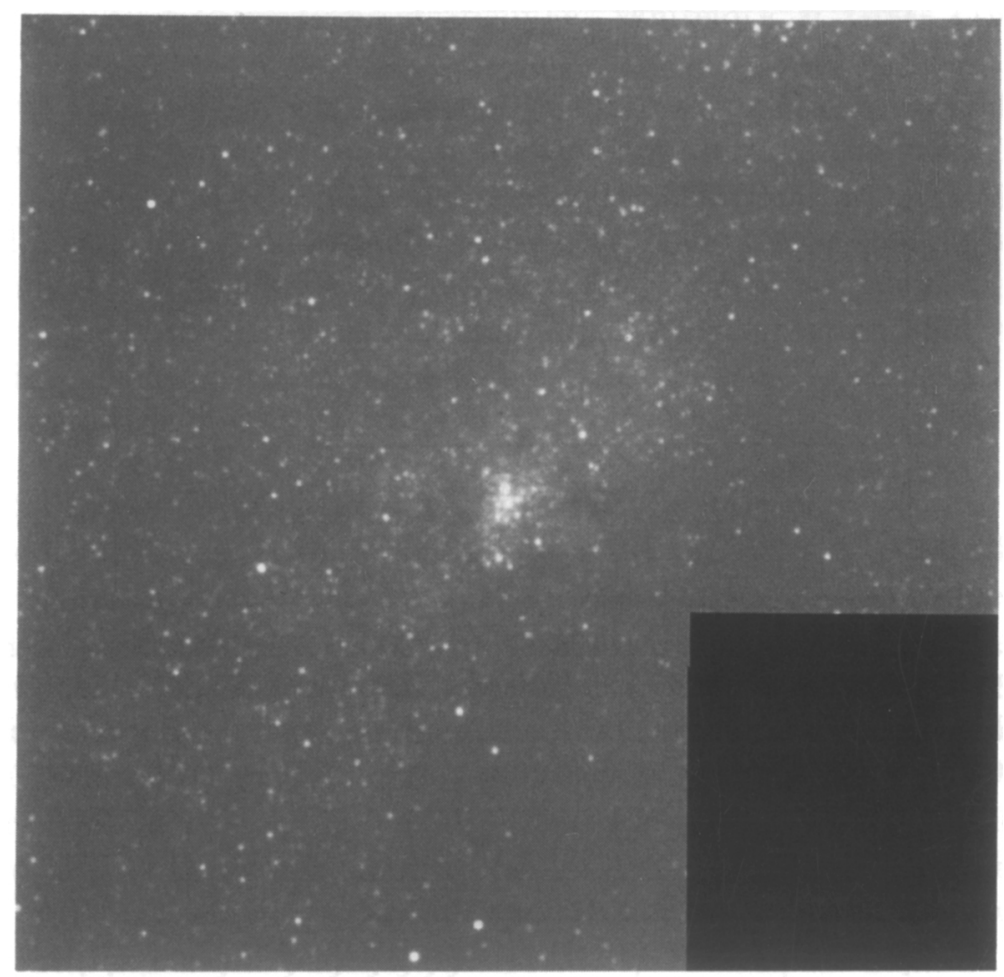

Fig. 3. Infrared image of the Galactic center in the $\mathrm{K}(2.2 \mu \mathrm{m})$ band by Haller $\&$ Rieke (1993); $6^{\prime}=12 \mathrm{pc}$ on a side, $\mathrm{N}$ at top, $\mathrm{E}$ to the right. The luminosity function in Figure 4 is drawn from this sample.

finds this population in fields $8^{\prime}$ perpendicular to the plane, where one has continuity with the surveys described below.

\section{The Inner 200 Parsecs}

The techniques of Stebbins \& Whitford were fundamentally sound, and had a detector of sufficient sensitivity been available, they could have mapped the central population and discovered the nucleus. An infrared map covering a $1 \mathrm{deg} \times 2 \mathrm{deg}$ field of the center was constructed from DC drift scans of a specially constructed InSb photometer (Glass, Catchpole, \& Whitelock, 1987). 


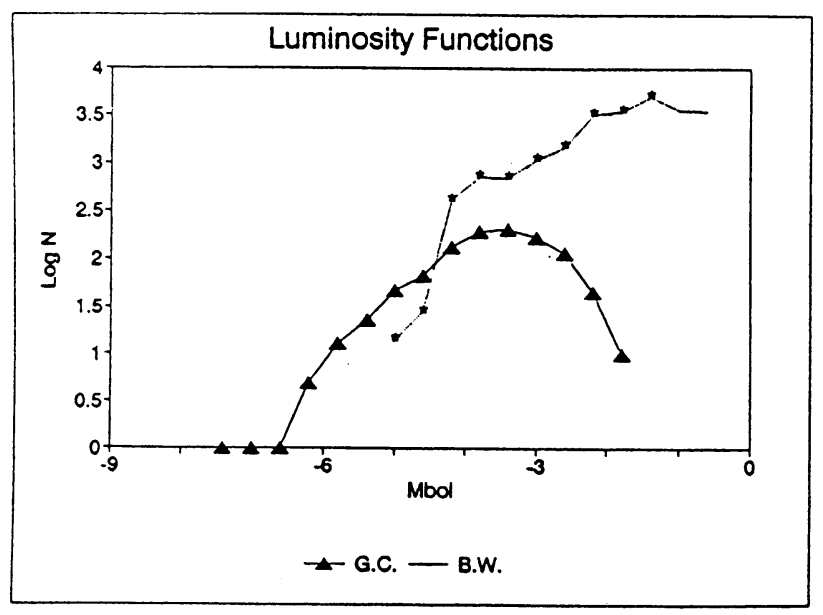

Fig. 4 (a). Luminosity function of late-type giants in the Galactic center field (Figure 3) triangles, and of optically selected $M$ giants in Baade's Window; Frogel \& Whitford, 1987, asterisks. Both are for $R_{0}=7 \mathrm{kpc}$; the Baade's Window luminosity function is scaled to the luminosity of the Galactic center. From Haller (1992) by permission. Notice the similarity of the Galactic center luminosity function to that of the M31 bulge (Figure 7).

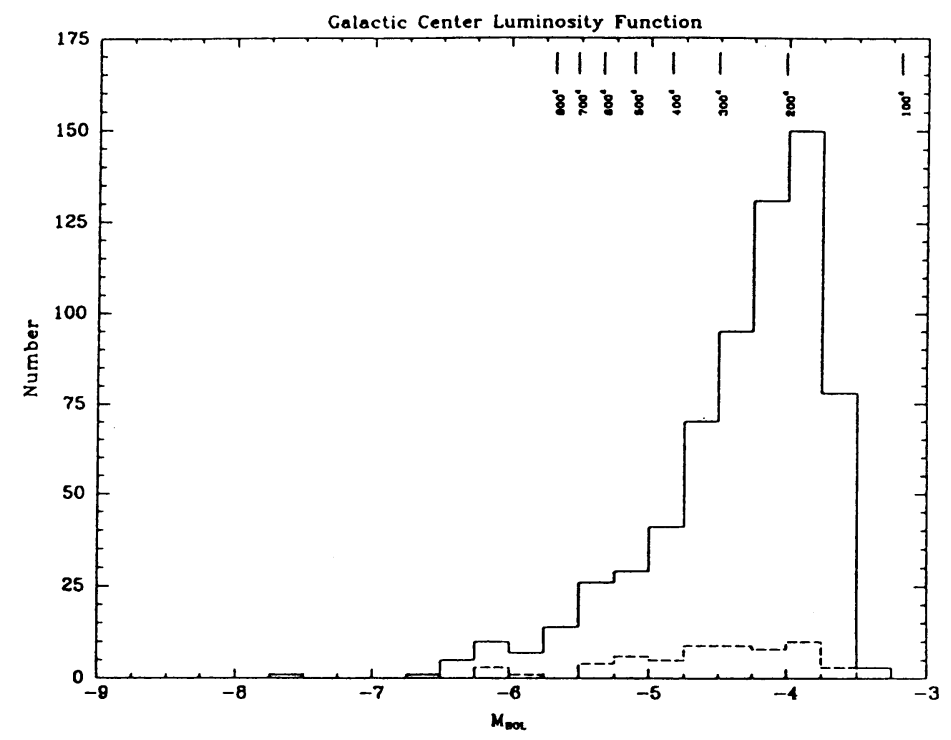

Fig. 4 (b). Bolometric luminosity function for 659 stars in the Haller (1992) Galactic center sample $m_{K}<11.0,(H-K)>1.50$. Solid histogram shows the total distribution while the dashed histogram is for the LPV candidates with $\delta m_{K}>0.27 \mathrm{mag}$. Periods at top are from the period-luminosity relationship for LMC and Galactic Miras; bulge Miras may be $\approx 0.5$ mag fainter at a given period (Whitelock, 1992, private communication). 
Analyzing these scans, Catchpole, Whitelock \& Glass (1990) find that the brightest stars $\left(M_{b o l}<-5.5\right)$ have a spatial distribution that is both flattened and concentrated to the center relative the bulk of the bulge. The luminosity function changes approaching the center. Figure $5 \mathrm{a}$ illustrates the flattened isophotes of the distribution, while $5 \mathrm{~b}$ shows the different luminosity functions. We are dealing here with a population extending $\approx 100 \mathrm{pc}$ out of the plane, and clearly not highly flattened. It is difficult to attribute this structure to a population that has been forming much mass within the last $2 \mathrm{Gyr}$, as some of the most luminous stars might require.

\subsection{STELLAR CONTENT AND STRUCTURE}

Is the central population dominated by the products of relatively recent $(<2 \mathrm{Gyr})$ star formation, or is it the metal rich population formed during the final stages of dissipative collapse $\approx 10 \mathrm{Gyr}$ ago? The luminous population favors the star formation hypothesis, but it is noteworthy that the luminous stars have a scale height much larger than $20 \mathrm{pc}$. Armed with the Catchpole et al. results, we can certainly argue continuity between the central $10 \mathrm{pc}$ and the next $100 \mathrm{pc}$. Very luminous stars such as IRS 7 and the He I cluster remain the exception rather than the rule.

Lindquist, Habing \& Winnberg (1992); LHW, report radial velocities and shell expansion velocities for $134 \mathrm{OH} / \mathrm{IR}$ stars close to the Galactic center. LHW's group II (high velocity outflow) OH/IR stars rotate $\approx 10$ times faster than the bulge stellar population and are no more than $30 \mathrm{pc}$ from the nucleus. Divided into low and high velocity expansion groups, $\mathrm{OH} / \mathrm{IR}$ stars show the classic dichotomy of the higher expansion velocity (younger? more metal rich?) group have lower velocity dispersion, higher rotation, and more concentration to the plane and nucleus. It is interesting that while LHW make their group I/II cut at $18 \mathrm{~km} / \mathrm{sec}$, van Langevelde (1990) makes a cut at $14.5 \mathrm{~km} / \mathrm{sec}$ and finds the same dichotomy, except this time over scales of $\approx 1000 \mathrm{pc}$, with rotation for his $\mathrm{OH} / \mathrm{IR}$ stars following that of the general stellar population (cf. 100 $\mathrm{km} / \mathrm{sec} / \mathrm{deg}$ ).

It is well known that abundances and kinematics are correlated in the K giants at $500 \mathrm{pc}$ (Rich, 1990). Sadler, Terndrup, and Rich (1993) are analyzing a sample of 400 bulge $\mathrm{K}$ giants with line strengths, radial, and proper motion velocity dispersions, and find that stronger lined stars have smaller vertical and radial velocity dispersions. Minniti (1993) finds greater rotation for the metal rich stars. The observed correlations between abundances and proper motion dispersions are almost certainly due to the increased rotation speed for the metal rich stars; the metal 


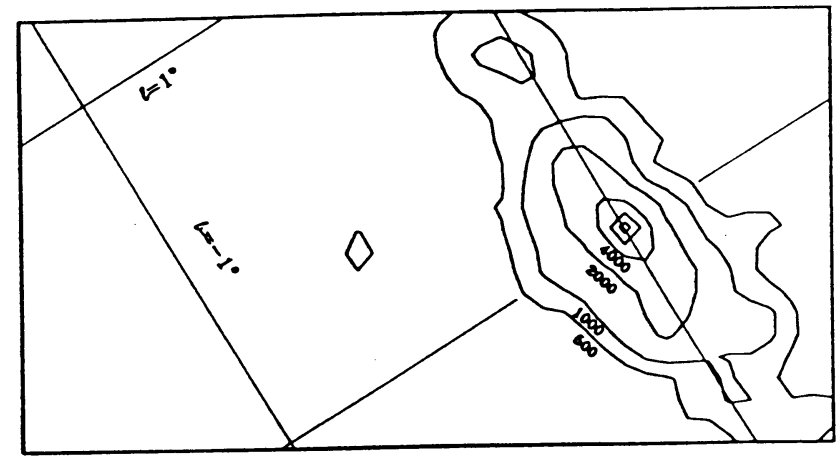

Fig. 5 (a). Contour map of the brightest stars $\left(5<K<6=-6<M_{\text {bol }}<-5\right)$ in the sample of Catchpole, Whitelock, \& Glass (1990). The K magnitude quoted is dereddened. Contours are marked by the number of stars per square degree. Note the flattened elliptical distribution centered on the Galactic Center (by permission).

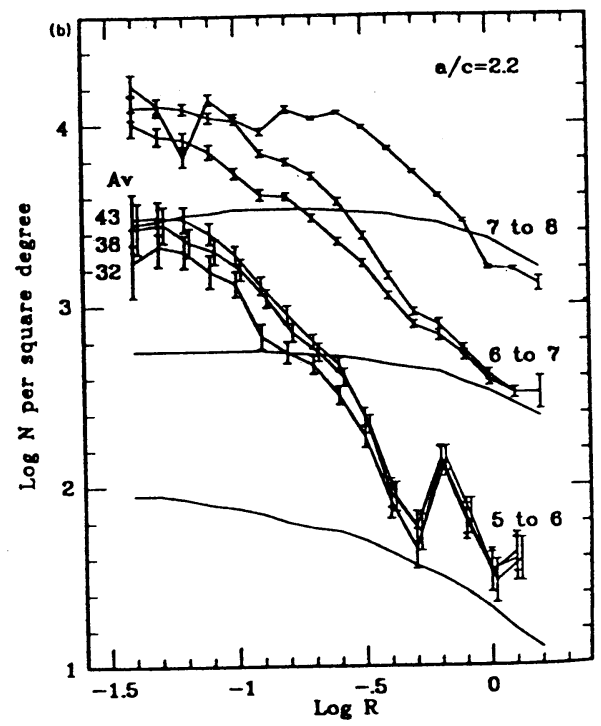

Fig. 5 (b). The concentration of the most luminous stars toward the Galactic center is evident (Catchpole, Whitelock \& Feast, 1990). The concentration of stars with $M_{b o l}<-5$ takes place over a scale of 100 pc. Number of stars per sq. deg shown as a function of $\log R$, angular distance in deg. from the Galactic center. Smooth curve are disk models for each luminosity class; because $K=7-8$ stars are seen only to 32 mag of visual extinction, 3 sets of counts were made in order to make certain the counts are comparable. 
rich stars must also have a more flattened spatial distribution (Figure 6; Zhao, Applegate \& Rich, 1992).

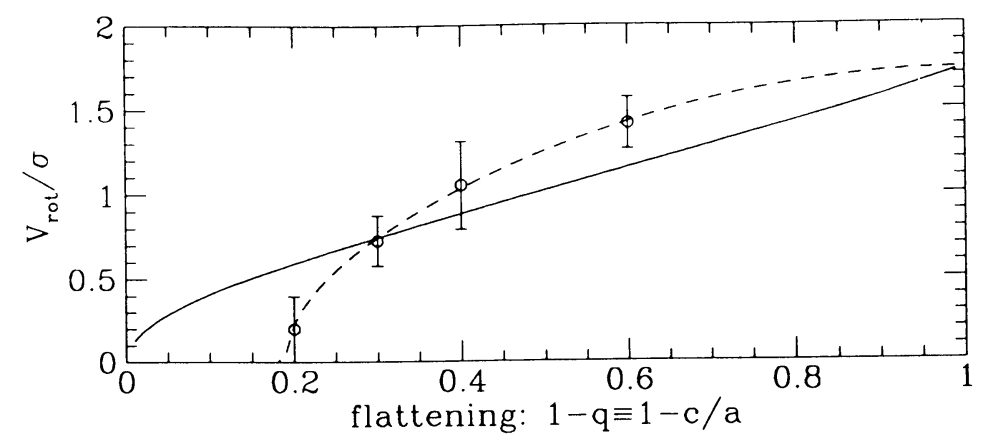

Fig. 6. Proper motion data in the bulge are consistent with more metal rich stars having a faster rotating, more flattened distribution than the general population. This possibly favors an enrichment and spin-up formation scenario which would lead to a super metal rich flattened population in the central 200 pc. Data points derived from Spaenhauer, Jones, and Whitford (1992) and Sadler, Terndrup, and Rich (1993). More metal rich stars are indicated with a larger value of flattening q. Solid line indicates a series of Maclaurin oblate rotator models, while the dashed line treats metallicity subgroups as massless tracers. Rotation velocity is derived from the assumption that observed proper motion anisotropy can be understood in the context of a flattened oblate rotator model for the bulge (Zhao, Applegate, and Rich, 1993).

There is no evidence for a highly flattened stellar system or discontinuity in stellar properties other than the obvious He I star cluster in the central few parsecs. Stars with $M_{b o l}<-5$ are concentrated to the nucleus; however such luminous stars are seen in the bulges of M31, M32 and M33. It is not clear at this time whether these luminous giants are intermediate age or metal rich.

3.1.1. Effect of Triaxiality. There is growing evidence that the bulge is a triaxial, possibly barred structure (Blitz \& Spergel, 1992; Binney et al. 1991; Whitelock \& Catchpole, 1992). Whitelock \& Catchpole's study is particularly compelling because of its use of the IRAS selected Miras to reveal a clear asymmetry as a function of Galactic longitude. As Spergel (1992) points out, triaxial potentials permit a class of orbits that intersect at the nucleus. It is possible that this orbit family is particularly effective at delivering gas to the center and fuels some of the activity. This may 
partially account for why the Milky Way appears to be more active than $\mathrm{M} 31$, and may have supported some star formation in the central $100 \mathrm{pc}$. The molecular gas occupies "forbidden" regions in the $l-V$ diagram that virtually require a triaxial potential (Binney et al. 1991).

\subsection{INTERSTELLAR MEDIUM}

Gas properties in the central $100 \mathrm{pc}$ are the topic of full papers, addressing both the dynamics and physical properties. Some $10 \%\left(5 \times 10^{8} M_{\odot}\right)$ of the Galaxy's molecular gas is in the center; this is in pressure equilibrium with $10^{8} \mathrm{~K}$ X-ray gas (Yamauchi et al. 1991). It is argued that while there is much molecular gas in the bulge region, high gas pressures (and consequent high internal velocity dispersions) are particularly unfavorable for star formation (Spergel \& Blitz, 1992; Morris, 1992).

In this volume, Whitford suggests that nature knows how to make stars in this region; he proposes that the rapidly rotating group of high outflow velocity $\mathrm{OH} / \mathrm{IR}$ stars were formed in this region further proposing that the disk continues within the central $100 \mathrm{pc}$. If this is so, how is it that the molecular gas in the middle of the Galactic potential lies so precisely in the Galactic plane? Is there any evidence for star formation in the plane outside the central parsec? Given the gap in HI from 1 to $3 \mathrm{kpc}$ (Burton \& Gordon, 1978) can one reasonably connect the Galactic center HI with the general disk?

\section{Bright Stars in Local Group Spheroids: A Distant Mirror}

Infrared arrays have recently been used to image the bulges of M31, M32, and M33, with the consequent discovery of luminous giants in these populations. Figure 7 shows the first such discovery (Rich \& Mould, 1991), that giants in a field $500 \mathrm{pc}$ from the nucleus greatly exceeded the $M_{b o l}=-$ 4.2 considered to be the AGB tip in the original Frogel \& Whitford (1987) luminosity function. While adoption of an $8 \mathrm{kpc}$ distance to the center effectively brightens this by $0.3 \mathrm{mag}$, the FW87 luminosity function still lacks bright stars. Figure 8 shows resolution of a field $2^{\prime}$ from the nucleus of M31; bright AGB stars are now also seen in M32 (Freedman, 1992a,b; Elston \& Silva, 1992). Using Kent's (1989) surface brightness model for M31, we can rule out the possibility that the bright stars belong to the M31 disk, as was suggested by Davies et al. (1991). Figure 9 shows the latest discovery, an $r^{1 / 4}$-law bulge population of $M_{b o l}=-6$ stars in M33 (Minniti \& Rieke et al. 1993).

In this volume, Renzini (his Table 1) points out that observers have been attempting photometry on stellar populations of dangerously high 


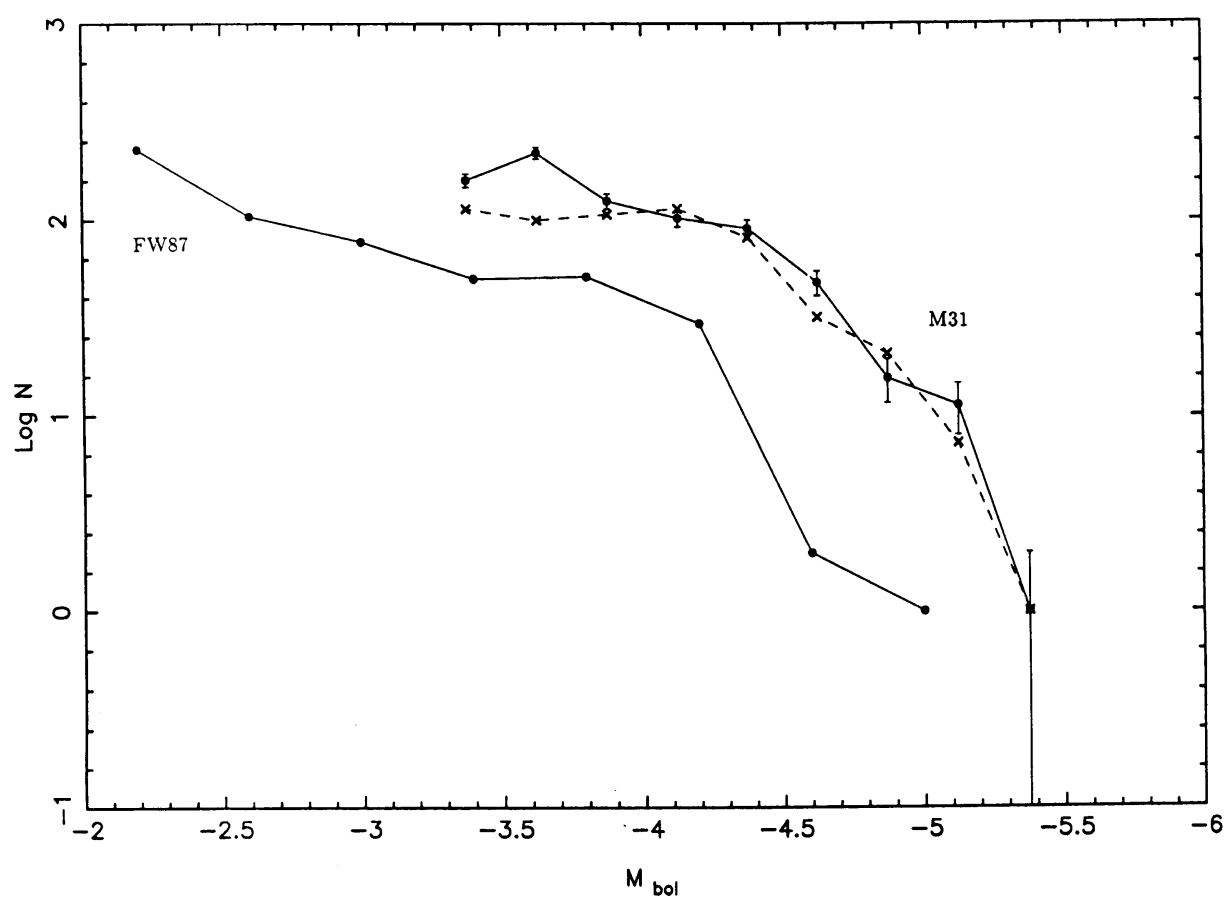

Fig. 7. Bolometric luminosity functions for a bulge field $500 \mathrm{pc}$ SE of the M31 nucleus (Rich \& Mould, 1991) and for Baade's Window (Frogel \& Whitford, 1987). The FW87 luminosity function is for $R_{0}=7 \mathrm{kpc}$; the assumed modulus to M31 is 24.2 . Currently favored distance moduli are 0.2 larger for both systems. Notice that the M31 luminosity function is extended to $M_{b o l}=-5$. Rich, Mould, \& Graham (1992) find that fields closer to the M31 nucleus may have even brighter stars and that these stars are not disk members (Figure 8).

surface brightness: some of my M31 fields have total luminosities of $10,000 L_{\odot}$ per sq arcsec. Is it possible that many of these "bright stars" are actually blended images, measured as a single star? I offer 3 arguments in support of my published photometry. First, Figures 8 and 9 both have $\approx 5,000 L_{\odot}$ per sq arcsec and are clearly resolved. Elston \& Silva (1992) publish an image centered $3^{\prime} \mathrm{E}$ of M32, where there is $500 L_{\odot}$ per sq arcsec. The field is notably uncrowded, and one could easily increase the star density by a factor of 10 and still do photometry. Finally, one can perform sensitive artificial star tests on these fields covering a wide range 


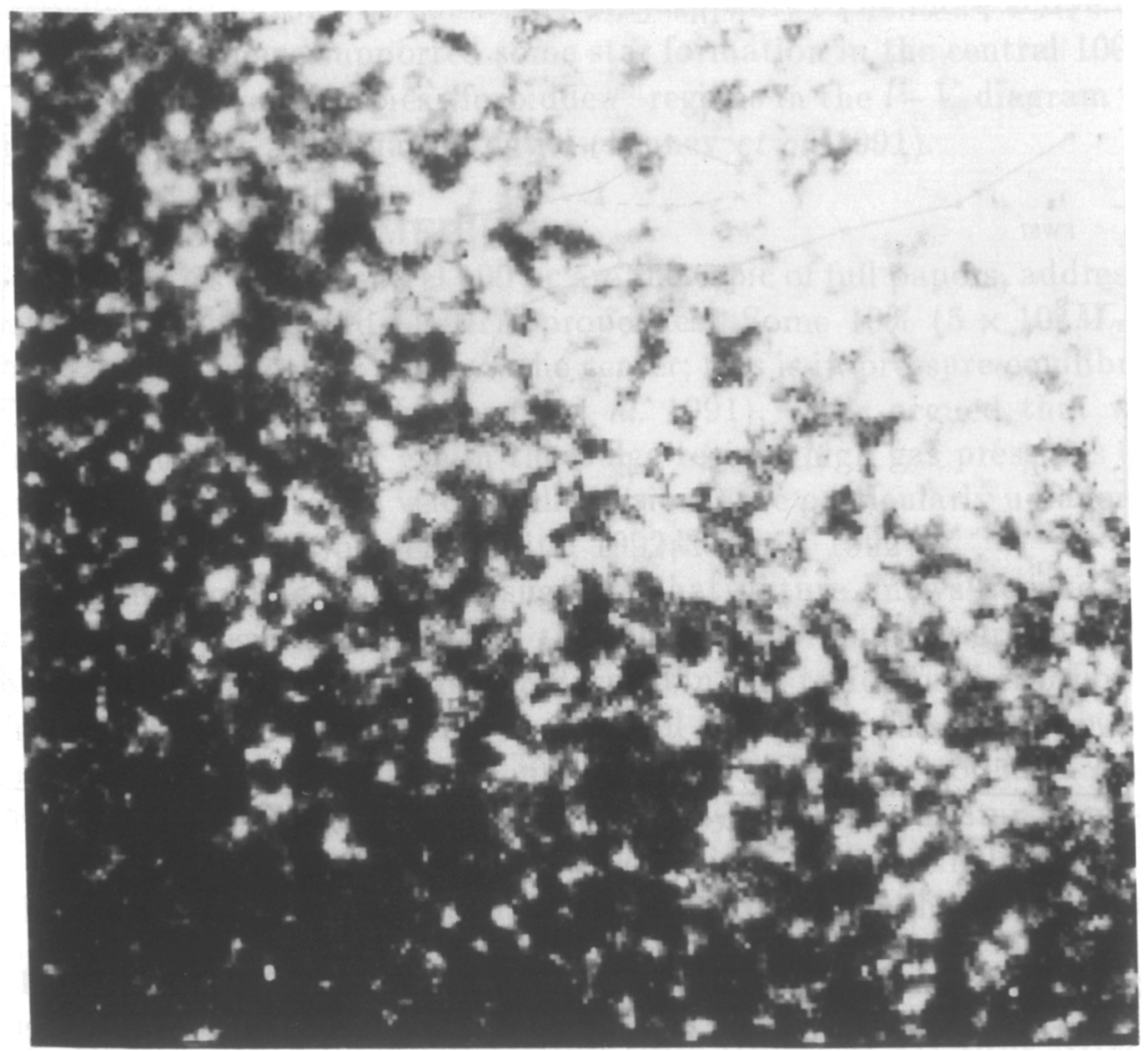

Fig. 8. $2 \mu \mathrm{m}$ image of the M31 bulge, $2^{\prime} \mathrm{SW}$ of the nucleus. North at top, East to right, $75 "$ on a side. The lower left edge of the field has $L_{T O T} \approx 10,000 L_{\odot}$ per sq. arcsec. Obtained with the Palomar infrared imager at the 4-m telescope (Rich; Mould \& Graham, 1992).

in surface brightness. Rich, Mould \& Graham (1992) observe M31 disk and bulge fields less than $1000 \mathrm{pc}$ from the nucleus. The artificial star tests find the innermost field ( $2^{\prime} \mathrm{SE}$ of the nucleus) to be overcrowded, but the other fields are relatively well behaved. Note that the luminosity functions of 2 different M32 fields are indistinguishable from the Rich \& Mould (1991) luminosity function illustrated in Figure 7. The extension 


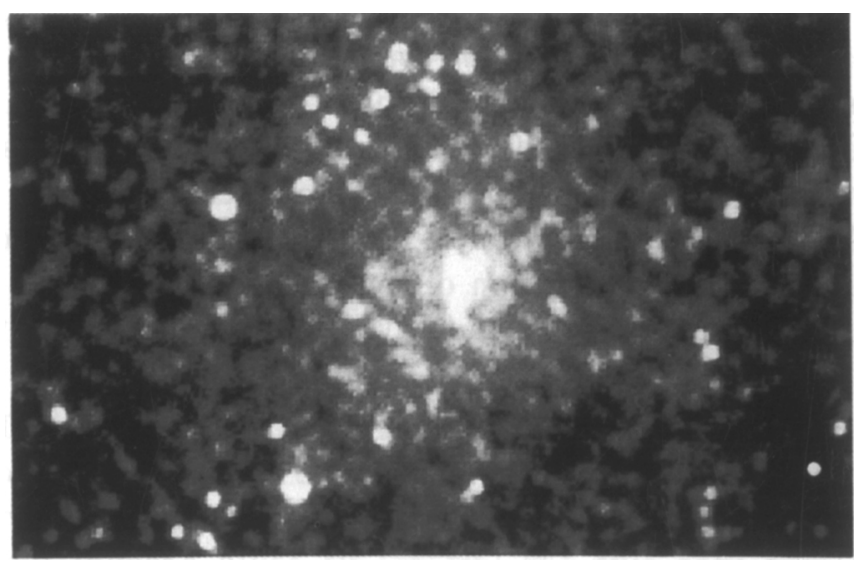

Fig. 9. In addition to M32, M31, and NGC 205, M33 joins the club of luminous bulge populations. This infrared frame by Minniti, Rieke et al. (1992) reveals an $r^{1 / 4}$-law distributed luminous stellar population of AGB stars with $M_{b o l}$ $=-6$. Image is $2.5^{\prime}$ in length; North at top, East to left. This is part of a $10^{\prime}$ long mosaic which also samples the disk of M33. While the surface brightness is $2000 L_{\odot}$ per sq arcsec, notice the clear resolution into stars.

to $M_{b o l}=-5.5$ is similar to that seen in the complete infrared surveys of Baade's Window reported by Glass et al. and Whitelock in this volume. Further, applying the Fuel Consumption Theorem one finds the the counts of the brightest stars per unit luminosity consistent with lifetimes of $\approx 10^{5} \mathrm{yr}$, or that of Miras. Despite a factor of 10 range in surface brightness, all of the bright stars in the local group populations studied so far have this approximate lifetime.

\section{What is the Central Population?}

Once we compare the population of the central $200 \mathrm{pc}$ with that of the M31 bulge and the surveys of Glass and Whitelock described in this volume, we no longer find the stars anomalously luminous. Even in the central $5 \mathrm{pc}$, where it might be argued that some star formation must have occurred, the luminosity function is only slightly brighter than is found in the central $500 \mathrm{pc}$ of the M31 bulge. Colors and luminosities of the stars are similar Whitelock et al. 's (1991) IRAS-selected Miras in the outer bulge. Glass et al. (1990) do not publish a luminosity function for 
the central $100 \mathrm{pc}$, but find a considerable number of stars in the interval $-5<M_{b o l}<-6$ remain even after correction for the disk. Bloemmaert (this volume) finds $\mathrm{OH} / \mathrm{IR}$ stars brighter than $M_{b o l}=-5$ in the outer bulge. As Catchpole et al. (1990) beautifully illustrate, there is no abrupt change in the central $200 \mathrm{pc}$, but a gradual increase in the number of luminous AGB stars, a trend which Haller continues to the central few pc. The remarkably high rotation of the central $\mathrm{OH} / \mathrm{IR}$ star population remains as the strongest evidence for young stars outside of the central parsec.

We have the problem of explaining whether the central $10^{8} M_{\odot}$ has formed in the last few Gyr or is largely the remnant of a more ancient event in which the most of the bulge formed. A star formation rate of $0.02 M_{\odot}$ per year over the last 4 Gyr could account for all of the mass. On the other hand, it is also possible that the concentration of high luminosity stars toward the center are the AGB progeny of a very metal rich ancient population. The observed flattening may relate to the collapse and spin-up inferred from the correlations between abundances and kinematics observed in the outer bulge; the central 200 pc may be mostly the result of the final stage of a dissipative collapse.

In this volume, Baum et al. report a bright main sequence turnoff at Baade's Window, confirmed by Ortolani \& Rich (1993). If the bulge was formed in a starburst $10 \mathrm{Gyr}$ ago (Rich, 1992) it is relatively easy to explain the high AGB luminosities. If the starburst formed a massive stellar disk (unstable to bar formation) then a thick bulge could have formed via the mechanisms described herein by Sellwood and Norman. The high luminosities and mass loss rates of the AGB stars still pose (in my opinion) a daunting challenge to those who would like the bulge to be the oldest population in the Galaxy. The key to learning the history of the central population is to understand the nature of the luminous AGB stars found in the Galactic bulge and other local group spheroids.

The effort to define the nature and origin of this central population precisely mirrors our struggle at this meeting to define what the bulges of galaxies are, and how they form. We are unsure whether they best represent disk-like structures, continuation of the spheroid, or a relative of the thick disk. In the spirit of this delightful confusion, I close this contribution with a humorous figure, and my apologies to the Belgian artist René Magritte.

I am grateful for Kris Sellgren's insights into this subject. I also acknowledge very lively discussions with Alvio Renzini on the topics of bulge formation and the nature of the AGB stars. I am grateful to Joe 


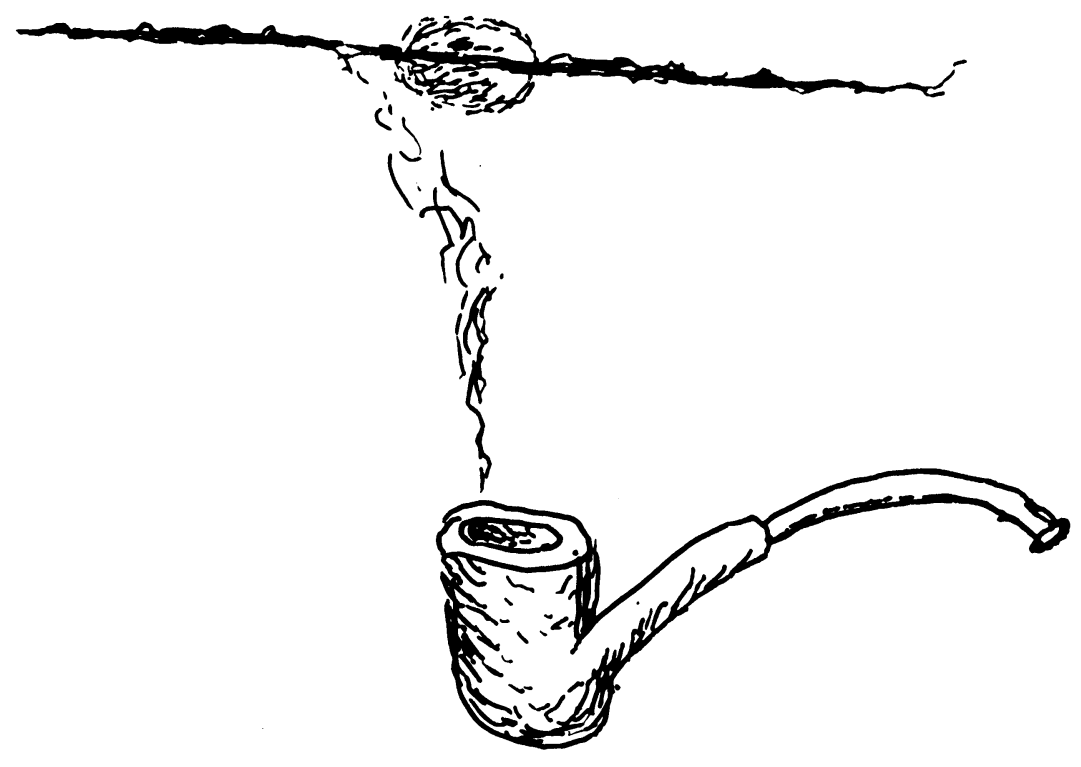

\title{
CECI NIEST PASUN BULBE
}

\author{
m. Rich \\ (APOLOGLES TO \\ RENIE MAGR(TTE)
}

Haller and Dante Minniti for their release of data prior to publication, and to Wendy Freedman and George Djorgovski for reviewing the manuscript. I am indebted to Francisco Feliciano for preparation of the manuscript.

\section{REFERENCES}

Allen, D.A., Hyland, A.R., \& Hillier, D.J. 1990, M.N.R.A.S., 244, 706. Backer, D.C., ed., The Galactic Center, AIP Conf. 155, (AIP:NY). Becklin, E., Gatley, I., and Werner, M.W. 1982, Ap.J., 258, 135.

Becklin, E.E., \& Neugebauer, G. 1968, Ap.J., 151, 145.

Binney, J. et al. 1991, M.N.R.A.S., 252, 210.

Blanco, V.M. 1965, in Vol. 5, Stars \& Stellar Systems, A. Blanco \& M. Schmidt, eds. (Univ. of Chicago Press, Chicago) p. 241. 
Blitz, L., ed., 1992, The Center, Bulge, and Disk of the Milky Way, (Dordrecht:Kluwer).

Blitz, L., \& Spergel, D.N. 1991, Ap.J., 379, 631.

Burton, W.B., \& Gordon, M.A. 1978, Astr. Ap., 63, 7.

Catchpole, R.M., Whitelock, P.A., \& Glass, I.S. 1990, M.N.R.A.S., 247, 479.

Davies, R.L., Frogel, J.A., \& Terndrup, D.M. 1991, A.J., 102, 1729.

Depoy, D., \& Sharp, N. 1991, A.J., 101, 1324.

Eckart, D. et al. 1992, Nature, 335, 526.

Elston, R., \& Silva, D.R. 1992, A.J., 104, 1360.

Feast, M. W. 1981, in Physical Process in Red Giants, A. Renzini \& I. Iben, eds. (Reidel: Dordrecht) p. 64.

Frogel, J.A. \& Whitford, A.E. 1987, Ap.J., 320, 199.

Freedman, W.L. 1992a, in IAU Symp. 149 The Stellar Populations of Galaxies B. Barbuy and A Renzini, eds. p. 169.

Freedman, W.L., 1992b, A.J., 104, 1349.

Gatley, I. in The Galactic Center, D.C. Backer, ed. AIP Conf. 155, (AIP:NY) p. 8.

Glass, I.S., Catchpole, R.M., \& Whitelock, P.A. 1987, M.N.R.A.S., 227, 373.

Kent, S.M.'1992, Ap.J., 387, 181.

Kent, S.M., Dame, T.M., \& Fazio, G. 1991 Ap.J., 378, 496.

Kent, S.M. 1989, A.J., 97, 1614.

Krabbe, A., Genzel, R., Dropatz, S., \& Rotaciuc, V. Ap.J. (Letters), 382, L19.

Lindqvist, M., Habing, H.J., \& Winnberg, 'A. 1992, Astr. Ap., 259, 118. Minniti, D.M. 1993, in preparation.

Minitti, D., Rieke, M. et al. 1993, Ap.J. (Letters), in press.

Morris, M., ed., 1989, The Center of the Galaxy, IAU Symp. 136 (Dordrecht:Kluwer) .

Morris, M. 1992, in preparation.

Ortolani, S., \& Rich, R.M. 1993, in preparation.

Renzini, A. \& Buzzoni, A. 1986 in Spectral Evolution of Galaxies, eds. C. Chiosi \& A. Renzini (Dordrecht: Reidel), p. 195.

Renzini, A., \& Fusi-Pecci, F.F. 1988 ARAA, 26, 199.

Rich, R.M., Mould, J.R., \& Graham, J. 1993, A.J., in press.

Rich, R. M. 1992, in The Center, Bulge, and Disk of the Milky Way L. Blitz, ed. (Reidel: Dordrecht) p. 47.

Rich, R.M. \& Mould, J.R. 1991, A.J., 101, 1286.

Rich, R.M. 1990, Ap.J., 362, 604. 
Sadler, E., Terndrup, D., \& Rich, R.M. 1993, in preparation.

Sellgren, K., McGinn,M.T., Becklin, E.E., \& Hall, D.N.B. 1990, Ap.J., 359, 112.

Sellgren, K. 1989, in The Center of the Galaxy, M. Morris, ed., IAU Symp. 136 (Dordrecht:Kluwer) p.477.

Spaenhauer, A., Jones, B., \& Whitford, A.E. 1991, A.J., 103, 297.

Spergel, D., \& Blitz, L. 1992, Nature, 357, 665.

Spergel, D. (1992) in The Center, Bulge, and Disk of the Milky Way L. Blitz, ed. (Reidel: Dordrecht) p. 77.

Stebbins, J., \& Whitford, A.E. 1947, Ap.J., 106, 235.

van der Veen, W., \& Habing, H.J. 1990, Astr. Ap., 231, 404.

van Herk, G. 1965, B.A.N., 18, 71.

van Langevelde, H. 1990, Ph.D. Thesis, Leiden University.

Whitelock, P. \& Catchpole, R. 1992, in The Center, Bulge, and Disk of the Milky Way L. Blitz, ed. (Reidel: Dordrecht) p. 103.

Whitelock, P.A., Feast, M.W., \& Catchpole, R.M. 1991, M.N.R.A.S., 248, 276.

Wood, P.R., \& Bessell, M.S. 1983, Ap.J., 265, 748.

Yamauchi, S. et al. 1991, Ap.J., 365, 532.

Yusef-Zadeh, F., \& Morris, M. 1991, Ap.J. (Letters), 371, L59.

Zhao, H.S., Applegate, J.H., \& Rich, R.M. 1993, in preparation. 


\section{DISCUSSION}

Tyson: Could you comment further on the highly flattened concentration of stars near the centre? In particular, are the flattened isophotes isolated, or do they blend smoothly with the general bulge?

Rich: It would be interesting to re-analyze the Catchpole work or push it with area detectors. They published this distribution of bright stars that have flattened ellipsoids at $\mathrm{K}=5$ to 6 , it would be interesting to look at the distributions of 6 to 7 and 7 to 8 at $\mathrm{K}$, and to see if there is any difference in the spatial distribution. Mould asserted (1986) that there is a concentration of bright stars towards the nucleus of M31.

Habing: We see a flattening, but that's on a slightly larger scale in the $\mathrm{OH} / \mathrm{IR}$ stars, where the high outflow velocities seem to be more concentrated to the plane. One way to find out whether the high outflow velocities are due to metallicity or to luminosity, is simply by obtaining the apparent luminosity of the stars.

Sellwood: The Winnberg and Lindqvist sample of $\mathrm{OH} / \mathrm{IR}$ stars have very distinct kinematics from the outer OH/IR sample. Is that telling us that the inner 200 parsec is something entirely different?

Rich: I think it is concentrated on a scale of about 100 parsec. I don't know because coexisting in that spatial volume are hotter populations. This particular sample has cold kinematics, there is no question of that.

Franx: No one has mentioned Tonry's work (on the luminosity variance method) in the last two days. I wonder if you can relate the result in our bulge and the M31 bulge to his work.

Rich: I think that what you see in the population depends sensitively on what wavelength you are working at. Tonry I believe is working in the $\mathrm{R}$ band and the luminosity function works very close to the centre, where it is calibrated, in M32. In the Virgo cluster one sees some reasonably correlation with what we see in M32, because you are looking at the fluctuations of the brightest giants in the $\mathrm{R}$ band. In the $\mathrm{K}$ band you start seeing rarer objects.

Tendrup: Tonry and I are talking about how to reconcile the bulge of M31 and other galaxies, in the brightness fluctuations method. If you do a simple calculation based on a bulge luminosity function, you get the same results as you do using M31 and M32. We plan to do this in the $\mathrm{K}$ band over the next year or so. 


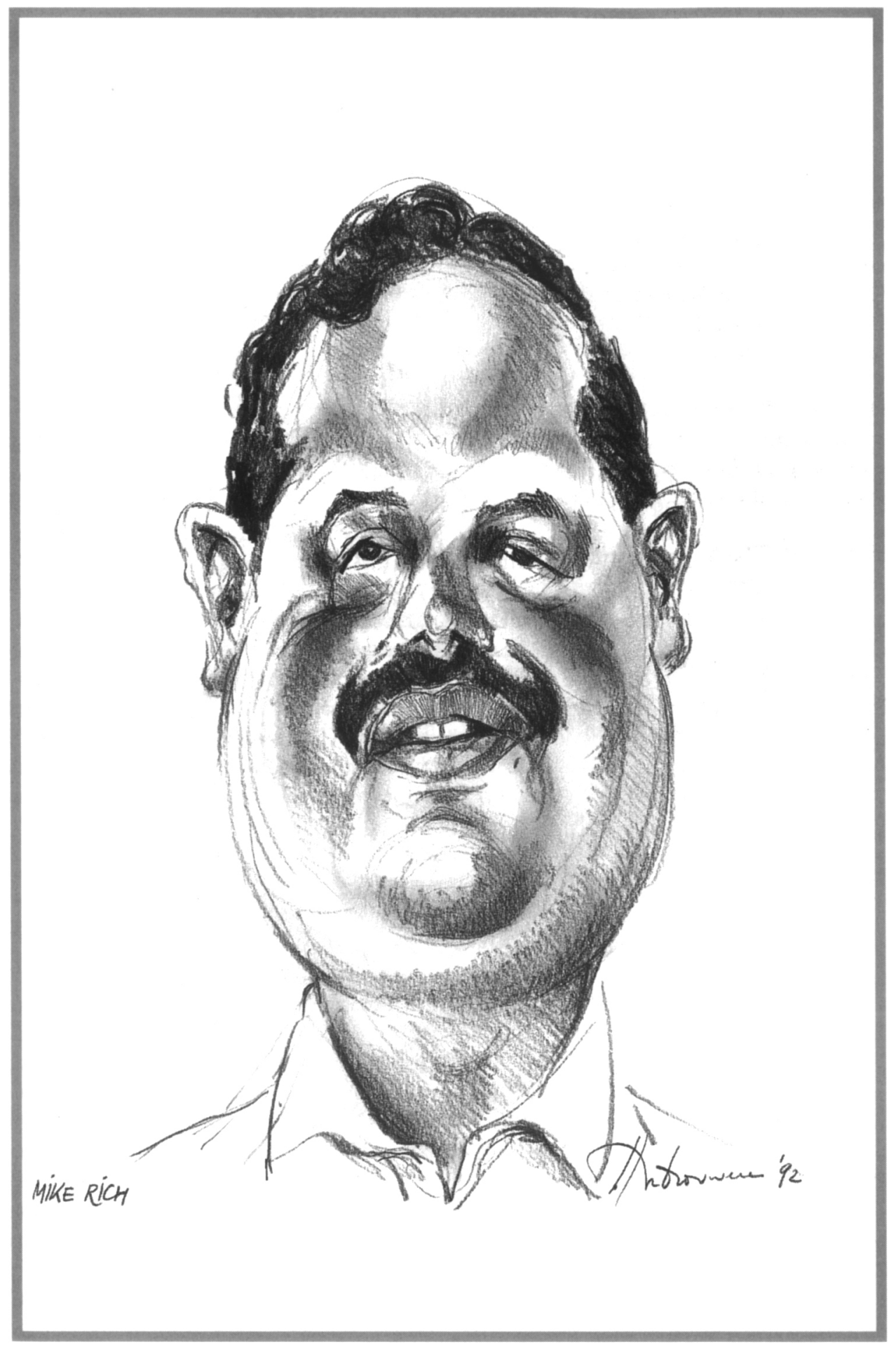




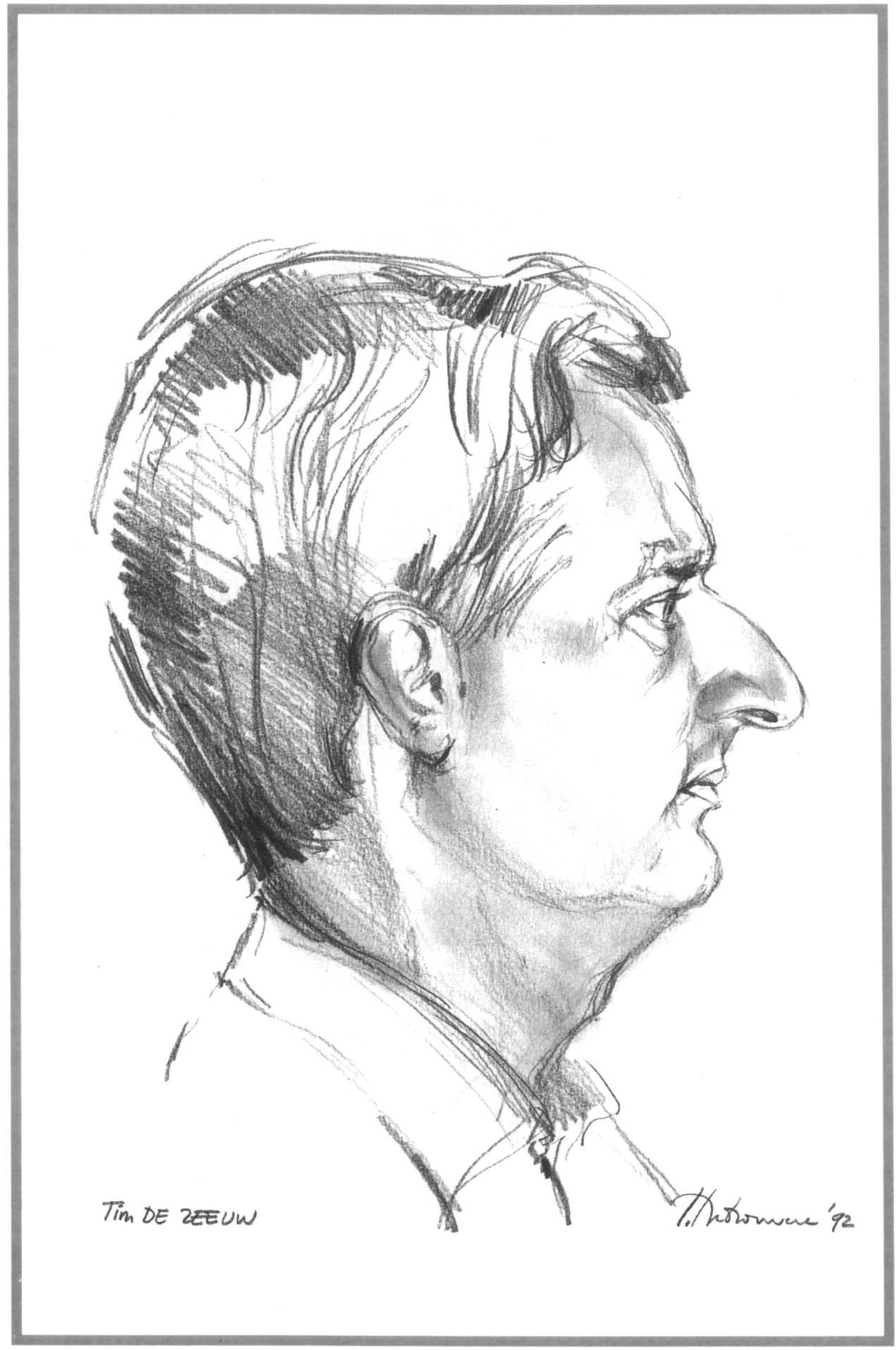

